\section{NMR Methods for the Investigation of Structure and Transport}

\author{
E.H. Hardy
}

New York, NY: Springer, 2011, 228 pages, $\$ 129$

The discoveries of nuclear MR (NMR) by I. Rabi, F. Bloch, and E. Purcell during World War II and of MR imaging by P. Lauterbur and P. Mansfield in the 1970s were major advancements in science, engineering, and medicine. Many NMR and MR imaging methods based on these discoveries have resulted in great practical benefits to society, and these methods continue to improve and to expand into new applications. A clear understanding of the fundamental principles and of some of the hardware and software requirements for optimally realizing these methods and applications is helpful for nearly every medical practitioner, scientist, and engineer.

This book is a compilation and summary of NMR- and MR imaging-related research and teaching at the author's institution, the Department of Chemical and Process Engineering of the Karlruhe Institute of Technology in Germany. The book is primarily intended for readers with an engineering background who are interested in applying or developing NMR or MR imaging methods for the study of structure and transport in mechanical, chemical, and food process engineering. However, the underlying principles and technical specifications of the methods are the same for medical applications such as anatomic, thermal, and blood flow imaging. Therefore, readers interested in clinical applications of these methods should also find the book relevant and useful.

The book comprises 9 chapters, but the main content of the book is contained in chapters 2 through 4 . Chapter 2 provides a fairly concise overview of the fundamentals of NMR and MR imaging processes. In addition, several basic and useful quantitative image analysis tools such as segmentation and phase correction are presented. Chapter 3 describes the major experimental components of an NMR system, including the magnets, gradient coils, and radiofrequency probes. Special emphasis is placed on the design of the actively shielded gradients and impedance matching of the RF probes. Chapter 4 describes some rather specific theoretic or experimental aspects of 10 distinct NMR- or MR imaging-related applications in various process engineering fields (e.g., gas filtration, solid-liquid separation, rheometry, structure of a ceramic sponge, flow and growth in a biofilm reactor, temperature mapping during microwave heating, and droplet size distribution in emulsions).

Wherever appropriate, the author has presented detailed mathematic equations and their derivations for better understanding of the quantitative aspects of the topics under discussion. A few sets of problems and solutions are provided in

COPYRIGHT (C) 2013 by the Society of Nuclear Medicine and Molecular Imaging, Inc.
Chapter 5, and the Matlab source codes for actual implementation of a few image processing algorithms are provided in Chapter 6. Readers (especially students or postdocs) who are keen to "get their hands dirty" should find this material helpful in testing and developing algorithms of their own for related problems.

\author{
Jingfei Ma \\ M.D. Anderson Cancer Center \\ 1400 Pressler St., Unit 1472 \\ Houston, TX 77030 \\ E-mail: jma@mdanderson.org
}

Published online Feb. 22, 2013.

DOI: $10.2967 /$ jnumed.113.120782

\section{Theranostics, Gallium-68, and Other Radionuclides: A Pathway to Personalized Diagnosis and Treatment}

R.P. Baum and F. Rösch, Eds.

New York, NY: Springer, 2013, 594 pages, $\$ 199$

The general themes of this book (theranostics and peptide receptor-targeted radiotherapy) derive from evolving successes in the use of generator-produced ${ }^{68} \mathrm{Ga}$ for molecular imaging of disease. The chapters represent an excellent synopsis of the scientific developments presented at the First World Congress on Gallium-68 and Peptide Receptor Targeted Radiation Therapy (June 2011), which attracted more than 300 investigators (from over 50 countries) to Bad Berka, Germany, for the scientific event.

The use of ${ }^{68} \mathrm{Ga}$ for molecular imaging of disease has seen a remarkable increase over the last several years. Applications for ${ }^{68} \mathrm{Ga}$ PET are emerging across a broad spectrum of diagnostic imaging challenges, including cancer, cardiovascular disease, infection, and inflammation. The increase in enthusiasm for ${ }^{68} \mathrm{Ga}$ use can be ascribed to several factors, including superiority in achievable image quality compared with other $\gamma$-emitting radionuclides (e.g., ${ }^{111} \mathrm{In}$ ); nuclear decay characteristics (i.e., half-life and positron emission branching ratio) that are considered favorable for clinical molecular imaging; and the potential for ondemand production via the introduction of maturing competitive generator and fluid-handling technologies that are now capable of providing a reliable, high-purity, on-demand ${ }^{68} \mathrm{Ga}$ precursor in sufficient quantities for routine radiopharmaceutical production in the absence of cyclotron operations. These characteristics promise an increasing role for ${ }^{68} \mathrm{Ga}$ as a tool for the design of 
compounds that have both diagnostic and therapeutic attributes (i.e., theranostics).

The book is organized into 4 sections. Part I details the history of ${ }^{68} \mathrm{Ga}$ generators and highlights recent advances in radiochemistry, generator technology, and fluid-handling systems that provide the basis to advance ${ }^{68} \mathrm{Ga}$ (and compounds labeled with therapeutic radionuclides, such as ${ }^{177} \mathrm{Lu}$ ) to clinical applications for patient care. Part II provides a broad review of the potential for ${ }^{68} \mathrm{Ga}$ and peptide receptor-targeted molecular imaging and radiotherapy through contributions highlighting the potential of ${ }^{68} \mathrm{Ga}$ for labeling of peptides, small molecules, nanoparticles, antibodies, and Affibody (Affibody AB) molecules. Part III focuses on clinical molecular imaging applications for ${ }^{68} \mathrm{Ga}$, with a heavy focus on the success of radiolabeled peptides targeted to the somatostatin subtype II receptor for imaging of neuroendocrine tumors, but also includes an interesting application of ${ }^{68} \mathrm{Ga}$ for lung perfusion imaging. Part IV provides excellent contributions highlighting recent success in peptide receptor radionuclide therapy for neuroendocrine tumors using ${ }^{177} \mathrm{Lu}$ and includes dosimetry contributions that can provide researchers with excellent background material needed to consider the introduction of new radiolabeled peptides for therapy and diagnostic imaging.

This book is an excellent resource for investigators interested in gaining a detailed understanding of the state of the art in ${ }^{68} \mathrm{Ga}$ and peptide receptor radionuclide therapy-based theranostics for preclinical and emerging clinical applications.

\author{
Michael K. Schultz, PhD \\ University of Iowa \\ ML B180 FRRB \\ 500 Newton $R d$. \\ Iowa City, IA 52242 \\ E-mail:michael-schultz@uiowa.edu
}

Published online Feb. 25, 2013.

DOI: 10.2967/jnumed.113.121350 